\title{
Depth in objective and subjective contour patterns
}

\author{
CLARE PORAC \\ University of Victoria, Victoria, British Columbia, Canada V8W 2 Y2
}

\begin{abstract}
Some evidence indicates that subjective contours arise when an observer organizes an ambiguous array into various depth planes because of implicit depth cues within the pattern. Two experiments were conducted to explore three-dimensional organization within subjective contour patterns. Although a size constancy scaling measurement technique produced data that were consistent with a depth hypothesis, more direct estimates of perceived depth produced contradictory evidence.
\end{abstract}

The perception of a visual contour usually requires the presence of an abrupt localized change in luminance or color. However, a number of patterns have been described in which an apparent contour is seen in the absence of an abrupt change in distal stimulation (Bradley \& Dumais, 1975; Coren, 1972; Gregory, 1972; Kanizsa, 1955, 1974, 1976; Schumann, 1904). These configurations are most often called subjective contours (Coren, 1972; Kanizsa, 1976) or cognitive contours (Bradley \& Dumais, 1975; Gregory, 1972; Kanizsa, 1974).

Several hypotheses have been advanced to explain the emergence of these apparent contours. Some suggest that they arise through lateral inhibitory interactions (Brigner \& Gallagher, 1974) or via the activation of cortical cells involved in contour formation and stimulus feature detection (Jung \& Spillman, 1970; Stadler \& Dieker, 1969, 1972). Alternatively, there are several cognitive explanations. For example, Coren (1972) suggests that observers utilize configurational depth cues such as interposition, texture gradients, and shadow to organize these patterns into a series of depth planes. The subjective contours then become the perceptual markers that indicate the boundaries of these various depth planes. Following this line of reasoning, the gaps in the four black circles in Figure 1(A) are organized as interruptions caused by a white square interposed in front of the four circles.

Although Coren's (1972) hypothesis of tridimensional organization is supported by some experimental evidence (Gregory \& Harris, 1974; Lawson, Cowan, Gibbs, \& Whitmore, 1974; Whitmore, Lawson, \& Kozora, 1976), there are several competing cognitive explanations. According to Gregory (1972), for instance, the subjective contour that is seen in Figure 1(A)

I would like to thank S. Coren for his helpful comments. In addition, I would like to acknowledge the assistance of Bonnie Robertson-Mann and the support of grants from the National Research Council of Canada (A0293) and from the University of Victoria. emerges as an "object hypothesis" to account for the gaps in the four black circles. Kanizsa $(1974,1976)$ and Schumann (1904) both favor closure, or figural completion tendencies, as the major organizational principle, while Kennedy $(1975,1976)$ indicates that configurational aspects of the stimulus array (such as perceived changes of direction) can be important in the emergence of subjective contours.

Kennedy $(1975,1976)$ has pointed out that subjective contour patterns elicit variable responses and impressions from observers. For this reason, it is difficult to collect data that are relevant to the issues raised by the various explanations of the phenomenon. Coren (1972) provided a technique that could allow one to investigate the relationship between depth and subjective contours. The technique involves an extrapolation from one of Coren's (1972) demonstrations. He observed that relative size distortions can occur because portions of the subjective contour array are perceived
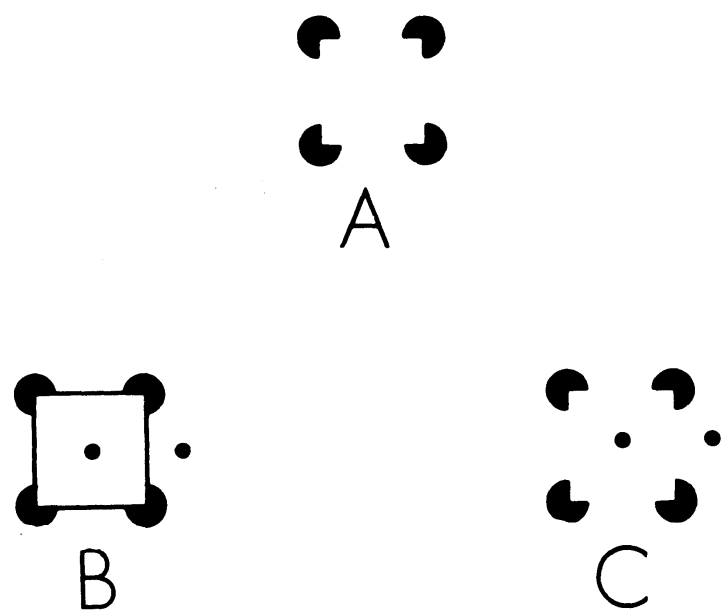

Figure 1. (A) An example of a subjective contour pattern in which a white square is seen in the absence of physically drawn contour lines. (B) Objective contour configuration used in Experiment 1. The outer test circle is shown in one of four possible positions. (C) Subjective contour configuration used in Experiment 1. 
to lie in various depth planes. Since a subjective contour figure is perceived as being in front of "background" portions of the pattern, a target imaged upon the apparently closer figure appears smaller than a target that is placed upon the apparent background. In other words, the depth processing involved in the formation of subjective contours is salient enough to evoke constancy scaling mechanisms when test targets are appropriately positioned upon the array. The test targets are processed as being at different distances from the observer, although they are actually equidistant. When size constancy scaling is inappropriately applied, the observer perceptually enlarges the apparently more distant target, resulting in a relative size distortion.

Although Coren (1972) reported some data that were consistent with his prediction of relative size distortion as a result of apparent depth, he did not provide an appropriate control comparison. He did not compare the potential misapplication of constancy scaling in subjective contour patterns, where illusory interposition cues are proposed to exist, with similar objective contour patterns, where interposition cues are physically present in the configuration. The purpose of Experiment 1 is to provide this missing control condition. It is proposed that a comparison of subjective and objective contour patterns employing this measurement technique will be useful in exploring the role of depth organization in the formation of subjective contours.

\section{EXPERIMENT 1}

\section{Method}

Subjects. One hundred and eighty-five adult volunteers participated in this study.

Stimuli. The stimulus configurations which were used are shown in Figure 1(B and C). The patterns were drawn in the center of a $20.2-\mathrm{cm}$ square of white paper. The sides of both the objective and the subjective contour squares were $2 \mathrm{~cm}$, the diameters of the four black inducing circles were $.8 \mathrm{~cm}$, and the diameters of the two test circles were $.25 \mathrm{~cm}$. The patterns were viewed from a distance of approximately $40 \mathrm{~cm}$. Under standard fluorescent room illumination, the reflectance of the black portions of each figure was approximately $4.1 \%$, while that of the white segments was $87 \%$.

Procedure. Ninety-two observers viewed the subjective contour pattern, while the remaining 93 participants made judgments concerning the objective contour figure. Each subject was shown one of the figures and was asked to make a relative judgment about the size of the test circles. Approximately one-half of the observers in each group was asked to indicate the larger of the two circles, while the remainder were instructed to choose the smaller of the two. Since many illusory phenomena tend to dissipate over time, all judgments were made within a 30-sec period. To control for any positional biases, the position of the outer or background test circle was randomly varied. It was to the right, to the left, above, or below the objective or the subjective square. The inner or figurally contained test circle always remained in the center of the pattern.

\section{Results and Discussion}

The data were analyzed by counting the number of responses that were consistent with the action of mis- applied constancy scaling within the figure. For example, if an observer was asked to choose the larger of the two test circles in either of the two patterns, and he or she chose the outer test circle as being the larger of the two, the choice was scored as consistent with the distortion that would arise from the misapplication of the constancy mechanism. Table 1 shows the frequency of these judgments for the subjective and the objective contour arrays.

There was a tendency for observers, when judging test elements within the subjective contour configuration, to make judgments consistent with the notion of misapplied constancy scaling as a result of apparent depth. This tendency did not appear among the observers who were exposed to the objective contour configuration. When tested, this difference was statistically significant $\left[\chi^{2}(1)=6.87, p<.01\right]$ and was consistent across all variations of stimulus presentation.

These findings replicate Coren's (1972) demonstration and seem to be consistent with his apparent depth explanation for the emergence of subjective contours. Since a depth cue (interposition) was theoretically present in both figures, it was interesting to find that the size distortion was confined to those observers who viewed the subjective contour figure. Perhaps this indicates phenomenal differences in the organization of depth relationships within the two patterns, which then affect the judgments of perceived size differences. This suggestion is tested in Experiment 2.

\section{EXPERIMENT 2}

\section{Method}

Subjects. Forty-one adult volunteers participated in this study. None of these individuals had participated in Experiment 1 .

Stimuli and Procedure. The two stimulus configurations were identical to those used in Experiment 1. However, the two test circles were removed from the patterns. Twenty-one observers viewed the objective contour figure, while the remaining 20 saw the subjective contour array. Upon viewing the figures, observers were asked to choose one of three statements that provided the best match to their own description of the figure. The three alternative descriptions were: (1) a white square in front of four black circles; (2) four black circles connected by lines; (3) four black circles with a wedge cut out of each one. One of the statements implies a three-dimensional perceptual organization, while the other two do not. This procedure is

Table 1

Judgments of Size Within Subjective and Objective Contour Patterns

\begin{tabular}{lccr}
\hline & \multicolumn{2}{c}{ Judgment of Size } & \\
& Consistent* & Inconsistent* & Total \\
\hline Subjective & 63 & 29 & 92 \\
Objective & 45 & 48 & 93 \\
Total & 108 & 77 & 185 \\
\hline
\end{tabular}

Note-Data expressed as number of observers. . * Consistent or inconsistent with the action of misapplied constancy scaling. 
Table 2

Phenomenal Descriptions of Objective and Subjective Contour Figures

\begin{tabular}{|c|c|c|c|c|}
\hline & \multicolumn{3}{|c|}{ Description } & \multirow[b]{3}{*}{ Total } \\
\hline & \multirow{2}{*}{$\frac{\text { Depth Implied }}{1}$} & \multicolumn{2}{|c|}{ No Depth Implied } & \\
\hline & & 2 & 3 & \\
\hline Subjective & 13 & 0 & 7 & 20 \\
\hline Objective & 20 & 0 & 1 & 21 \\
\hline Total & 33 & 0 & 8 & 41 \\
\hline
\end{tabular}

Note-Data expressed as number of observers.

analogous to one used by Kennedy (1975) to explore the possibility of apparent depth separation within portions of subjective contour figures.

\section{Results and Discussion}

Table 2 shows that apparent depth descriptions were given for both patterns. However, the differences in the size judgment findings of Experiment 1 do not appear to be related to differences in phenomenal depth organization. In this experiment, it was the objective and not the subjective contour figure that was more likely to be described as three-dimensional. When tested, this difference was statistically significant $\left[\chi^{2}(1)=5.96, p<.05\right]$. Thus, in contrast to what one might expect from the size judgment data, phenomenal depth organization tended to favor the objective array.

The results of Experiment 1 support the notion that depth separation exists within portions of subjective countour patterns. However, the findings from Experiment 2 indicate that observers may not consistently report apparent depth with these figures. Thus, the two techniques for assessing perceived depth have produced interesting asymmetries when objective and subjective contour figures are compared. Phenomenal reports of depth seemed to favor the objective contour pattern, whereas illusory size distortions, presumably related to depth separation, were found only in the subjective contour array. These findings have implications for the investigation of mechanisms operative in the formation of subjective contours. Not only do they point out that different measurement techniques may produce different results, but they also indicate that comparisons to objective contour patterns, where possible, may prove to be extremely useful. Further investigations will have to reveal whether these measurement asymmetries are based upon underlying differences in the processing of objective and subjective contour patterns.

\section{REFERENCES}

Bradley, D., \& Dumais, S. Ambiguous cognitive contours. Nature, 1975, 257, 582-584.

Brigner, W. L., \& Gallagher, M. B. Subjective contour: Apparent depth or simultaneous brightness contrast? Perceptual and Motor Skills, 1974, 38, 1047-1053.

Coren, S. Subjective contours and apparent depth. Psychological Review, 1972, 79, 359-367.

GrEgory, R. L. Cognitive contours. Nature, 1972, 238, 51-52.

GREgORY, R. L., \& HARRIS, J. P. Illusory contours and stereo depth. Perception \& Psychophysics, 1974, 15, 411-416.

Jung, R., \& Spillman, L. Receptive-field estimation and perceptual integration in human vision. In F. A. Young \& D. B. Lindsley (Eds.), Early experience and visual information processing in perceptual and reading disorders. Washington, D.C: National Academy of Science, 1970.

KanIzSA, G. Marzini-percettivi in campi con stimolazione omogenea. Rivista di Psicologia, 1955, 49, 7-30.

KANIZSA, G. Contours without gradients or cognitive contours. Italian Journal of Psychology, 1974, 1, 93-113.

Kanizs A, G. Subjective contours. Scientific American, 1976, 234, 48-52.

Kennedy, J. M. Depth at an edge, coplanarity, slant depth, change in direction and change in brightness in the production of subjective contours. Italian Journal of Psychology, $1975,2,107-123$.

Kennedy, J. M. Attention, brightness, and the constructive eye. In M. Henle (Ed.), Vision and artifact. New York: Springer, 1976. Pp. 33-48.

Lawson, R. B., Cowan, E., Gibbs, T. D., \& Whitmore, C. G. Stereoscopic enhancement and erasure of subjective contours. Journal of Experimental Psychology, 1974, 103, 1142-1146.

Schumann, F. Einige beobachtungen uber die zusammenfassung von gesichtseindrucken $\mathrm{zu}$ einheiten. Psychologische Studien, 1904, 1, 1-32.

Stadler, M., \& Dieker, J. Margini quasi-percettivi e aftereffects figurali. Rivista di Psicologia, 1969, 63, 95-104.

STADleR, M., \& Dieker, J. Untersuchungen zum problem virtueller konturen in der visuellen wahrnehmung. Zeitschrift fur Experimentelle und Angewandte Psychologie, 1972, 19, 325-350.

Whitmore, C. L. G., Lawson, R. B., \& Kozora, C. E. Subjective contours in stereoscopic space. Perception \& Psychophysics, 1976, 19, 211-213.

(Received for publication November 9, 1977.) 